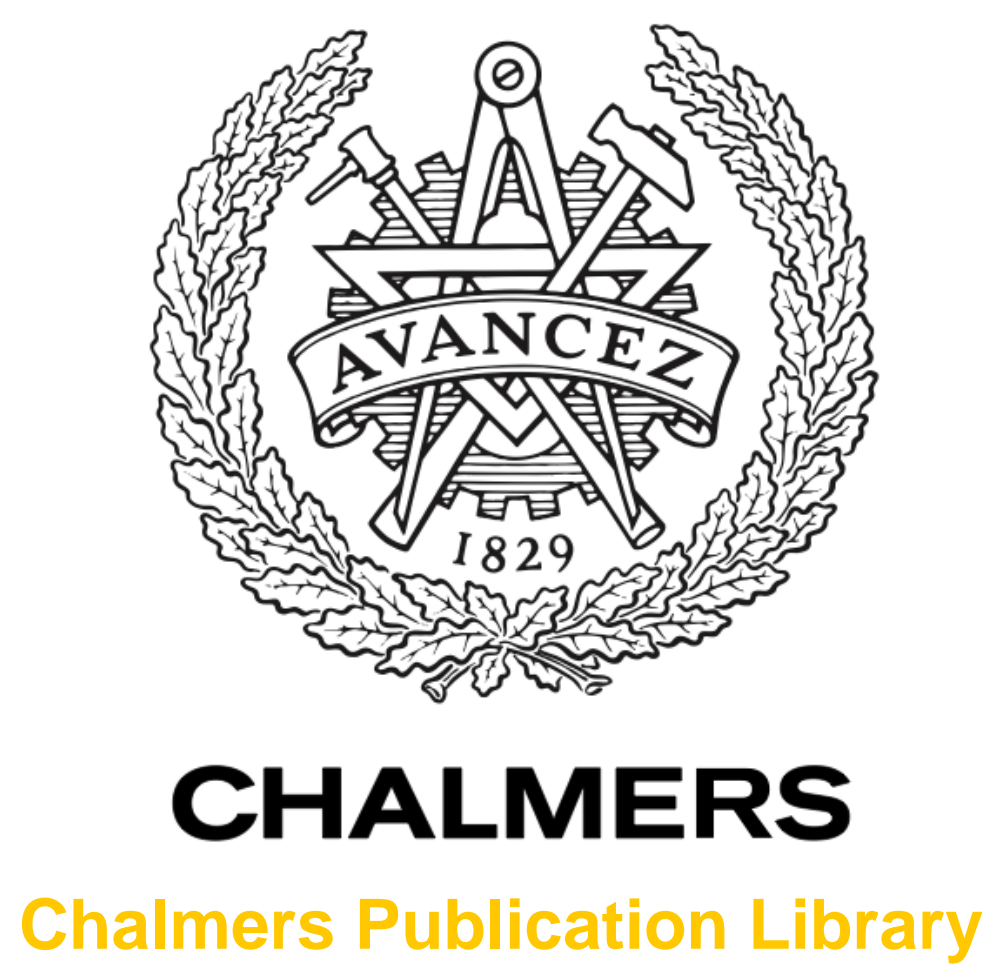

\title{
Array response interpolation and DOA estimation with array response decomposition
}

This document has been downloaded from Chalmers Publication Library (CPL). It is the author's version of a work that was accepted for publication in:

Signal Processing (ISSN: 0165-1684)

Citation for the published paper:

Yang, B. ; McKelvey, T. ; Viberg, M. et al. (2016) "Array response interpolation and DOA estimation with array response decomposition". Signal Processing, vol. 125 pp. 97-109.

http://dx.doi.org/10.1016/j.sigpro.2015.12.023

Downloaded from: http://publications.lib.chalmers.se/publication/235236

Notice: Changes introduced as a result of publishing processes such as copy-editing and formatting may not be reflected in this document. For a definitive version of this work, please refer to the published source. Please note that access to the published version might require a subscription. 


\title{
Array Response Interpolation and DOA Estimation
}

\section{with Array Response Decomposition}

\author{
Bin Yang $^{a, b, *}$, Mats Viberg ${ }^{b}$, Tomas McKelvey ${ }^{b}$ Guanghan Xu ${ }^{a}$ \\ ${ }^{a}$ School of Electronic and Information Engineering, Beihang University, Beijing 100191, China \\ ${ }^{\mathrm{b}}$ Department of Signals and Systems, Chalmers University of Technology, SE-412 96, Gothenburg, Sweden
}

\begin{abstract}
High-resolution direction-of-arrival (DOA) estimation requires an accurate array response model, which is usually achieved by measuring the response for given directions of the sources and employing interpolation. This paper considers the array response interpolation and DOA estimation by coherently combing parametric modeling and interpolation techniques. The proposed method is to decompose the array response as a product of a mutual coupling matrix, an ideal array response vector (dependent on the geometry of antenna array) and a DOA-dependent correction vector. Our idea is now that the correction vector will be a smoother function of DOA as compared to direct interpolation, since parts of the phase variation in one embedded element are due to the mutual coupling and the ideal array response. In numerical experiments of a real antenna array, the method is found to improve the performance of array response interpolation and DOA estimation significantly when the array response is updated by interpolation of the correction vector. In addition, we show how the DOA estimation performance can be predicted using a simple model of the interpolation error statistics.
\end{abstract}

Keywords: Direction-of-arrival (DOA) estimation; Array response interpolation; Correction vector; Parametric modeling.

\section{Introduction}

Direction of arrival (DOA) estimation uses the data received by an array antenna to estimate the direction of one or more signal sources. In an ideal data model, the array response can be determined using the geometry of the array [1]-[4]. For a real antenna array, it is well known that the array modeling errors can have a significant influence on DOA estimation if these effects are not correctly accounted for [5]-[8].

To remedy the array modeling errors, an interesting approach is to employ a parametric model of the array response, where array parameters are used to tune the model response to match measurements 1 
[9]-[17]. However, a crucial question is identifiability of the appropriate parameters. In [9]-[11], a parametric vector is used to model the uncertainty in the array response matrix. Examples of array compensation procedures using a diagonal array correction matrix are given in [12]-[14]. Both a parametric vector and a diagonal correction matrix can correct for channel errors only. An arbitrary full-rank correction matrix is employed in array calibration using either a local or global model in [15]-[17]. This can model both channel and mutual coupling errors. Further, a special structure of the mutual coupling matrix is proposed for a uniform linear array (ULA), a uniform hexagon array (UHA) and a uniform circular array (UCA), respectively [18]-[20]. In these parametric methods the performance is still found to be limited by the choice of parameterization, and the practical applicability is further limited by the increasing number of parameters. Eigenstructure based methods for joint estimation of the gain and phase of each sensor and DOA are presented in [21]-[22]. In [23], a similar approach is used to simultaneously estimate DOA and mutual coupling parameters. These methods are computationally efficient for the case of linear array parameters. However, the performance is limited by that of the MUSIC algorithm, and in particular the sources must be resolvable by MUSIC. Further, a more detailed physical model of the antenna array response will lead to a better match to real or measured data. However, no model regardless how complicated will ever be able to capture all possible effects. A simple method is to use measured data of the element far-field functions in the array antenna. However to enable accurate DOA estimation, the grid size must be selected rather small, which would require a large memory storage and a long data collection (or simulation) time. Based on the measured array response, another interesting approach that does not need specifying how the array response model depends on the array parameters is the array interpolation or manifold separation [24]-[28]. The idea is to use a Fourier representation of the array response in [24]-[26], which is based on the manifold separation technique (MST). This requires that the array response to be collected on a uniform grid covering the whole range. In [25]-[26], the array interpolation technique is successfully applied in combination with the element-space (ES) root-MUSIC algorithm. However, the method only directly uses the measured array response data, which requires a large number of Fourier terms and more memory to enable high-resolution DOA estimation. An alternative approach is to use local linear interpolation (LLI) of a factor of the array response model, which is often sufficient [27]-[28]. It does not require storing any special parametric data for array response calibration, such as the Fourier coefficients. The interesting contribution is that 
an ideal array response vector is decomposed from the measured (or simulated) response in these methods. However, a dense grid size is still required to enable high-resolution DOA estimation, since the mutual coupling captures parts of the phase and amplitude dependence on the array response in general.

An interesting alternative is to jointly use the smoothed interpolation of a correction vector (DOA-dependent) and mutual coupling matrix estimation. In this paper, we propose to use knowledge of the element positions and a simple model of the mutual coupling. A mutual coupling matrix, an ideal array response vector (ideal array steering vector) and a correction vector are decomposed from the measured array response in our method. The idea is now that the correction vector will then be a smoother function of DOA as compared to the original array response, since most of the phase-dependence in one embedded element is due to the mutual coupling and the ideal array response vector. First, a mutual coupling matrix is computed by the simulated (or measured in lab) array response as well as the isolated element response. The second step is to determine the correction vector at given directions using the measured array response and the estimated mutual coupling matrix. Third, either a Fourier representation or a linear interpolation is used to interpolate the correction vector instead of directly interpolating the array response. Finally, the array response interpolation is calculated by the interpolation of the correction vector, so that the number of Fourier terms can be kept as small as possible, or alternatively the interpolation grid size can be kept as small as possible. This also results in a more accurate interpolation and DOA estimation in a real antenna array, either for a given interpolation grid size or for a given number of Fourier terms.

The original contributions of this paper are as follow:

a) We show how an exploitation of a simple parametric model of the array response leads to a much smoother interpolation problem than using the original array data;

b) We exemplify the idea by applying a simple model of the mutual coupling that can be learned from the measured data. Note that it is not necessary for this simple model to be perfect for array response interpolation, since the mutual coupling model will be updated by a correction vector;

c): We show that a simple model of the resulting array interpolation error statistics is practically useful for predicting the performance of a DOA estimator.

This paper is organized as follows. In Section 2 we formulate the problem. Next, the basic mathematical models of DOA estimation are introduced in Section 3. Section 4 introduces the three 
interpolation methods of the array response including the mutual coupling estimation. A real array of 12 quadrifilar helix antennas (QHAs) is presented in Section 5, whereas Section 6 shows the results of the three interpolation methods when applied to the real antenna array. The different DOA estimation approaches are then compared in terms of applicability and performance in Section 7. The paper is finally concluded in Section 8 .

\section{Problem formulation}

Assume that there are $N$ antenna elements in the array located in the x-y plane, and $M$ far-field narrowband signal sources impinging on the array. The resulting signal at element $n$ with sample index $k$ is denoted $x_{n}(k)$, for $n=1, \cdots, N$. Then, the resulting data are collected in the array output vector $\mathbf{X}(k)=\left[x_{1}(k), \cdots, x_{N}(k)\right]^{T}$, which can be expressed as

$$
\mathbf{X}(k)=\sum_{m=1}^{M} \mathbf{a}_{e m b}\left(\eta_{m}\right) s_{m}(k)+\mathbf{W}(k)
$$

Here, $\eta_{m}$, which is characterized by the polar angle $\theta$ and the azimuth angle $\phi$ in a 3-dimensional space model, is termed the DOA of the $m$-th signal source, $\mathbf{a}_{e m b}(\eta)=\left[a_{e m b, 1}(\eta), \cdots, a_{e m b, N}(\eta)\right]^{T}$ is the array response to a signal from the DOA $\eta, s_{m}(k)$ is the corresponding signal waveform from the $m$-th signal source, and $\mathbf{W}(k)$ represents an additive noise term.

High-resolution DOA estimation requires that the functional form of the array response $\mathbf{a}_{e m b}(\eta)$ is accurately known. In an ideal uniform antenna array model case, the isolated element pattern is omnidirectional and no mutual coupling exists. The array response is obtained by $\mathbf{a}_{e m b}(\eta)=\mathbf{a}_{\text {ideal }}(\eta)$, where $\mathbf{a}_{\text {ideal }}(\eta)=\left[a_{\text {ideal }, 1}(\eta), \cdots, a_{\text {ideal }, N}(\eta)\right]^{T}$ is the ideal array response vector that comes from the geometry only. In real antenna array modeling, an element of the array response is obtained by measuring this element in the presence of all other elements, which takes the ideal array response vector $\mathbf{a}_{i \text { deal }}(\eta)$, mutual coupling and each isolated element properties into account [29]-[33]. This is the approach we follow here. Thus, it is assumed that this, so called embedded array response, has been measured at a grid of DOA values, $\eta_{1}, \eta_{2}, \cdots, \eta_{L}$, where $L$ is the number of measured point. With the aid of this calibration data and a simple model of the array response, the goal is to estimate the unknown DOAs in an estimation data set $x(k), k=1,2, \cdots, K$.

Assuming that the ideal steering vector $\mathbf{a}_{\text {ideal }}(\eta)$ is known, we have two basic models to decompose the array response. 
1) The first model of the embedded array response is

$$
\mathbf{a}_{e m b}(\eta)=\mathbf{a}_{\text {ideal }}(\eta) \odot \mathbf{g}(\eta)
$$

where $\odot$ denotes the Schur (Hadamard) product and $\mathbf{g}(\eta)=\left[g_{1}(\eta), \cdots, g_{N}(\eta)\right]^{T}$ is now the correction vector, which is dependent of the DOA $\eta$. It takes care of the effects of mutual coupling and the properties of the isolated elements.

2) The second model is

$$
\mathbf{a}_{e m b}(\eta)=\mathbf{C}\left[\mathbf{a}_{i \text { deal }}(\eta) \odot \mathbf{g}_{e}(\eta)\right]
$$

where $\mathrm{C}$ is the $N \times N$ mutual coupling matrix, which is independent of the DOA and $\mathbf{g}_{e}(\eta)=\left[g_{e, 1}(\eta), \cdots, g_{e, N}(\eta)\right]^{T}$ is the correction vector, which is dependent of the DOA $\eta$. Note that $\mathbf{g}_{e}(\eta)$ only holds the properties of the isolated element in this model, which is different from the $\mathbf{g}(\eta)$ in the first model.

Using an accurately measured array response at a given DOA grid $\eta_{l}, l=1,2, \cdots, L$, the correction vector $\mathbf{g}\left(\eta_{l}\right)$ in the first model can be determined as

$$
\mathbf{g}\left(\eta_{l}\right)=\mathbf{a}_{e m b}\left(\eta_{l}\right) \cdot / \mathbf{a}_{\text {ideal }}\left(\eta_{l}\right)
$$

where ./ denotes element-wise division. Given an interpolated $\hat{\mathrm{g}}(\eta)$ at a certain desired DOA $\eta$, the array response interpolation is then computed as

$$
\hat{\mathbf{a}}_{e m b}(\eta)=\mathbf{a}_{i d e a l}(\eta) \odot \hat{\mathbf{g}}(\eta)
$$

In addition, if the mutual coupling is known in the second model, the correction vector $\mathbf{g}_{e}\left(\eta_{l}\right)$ can be determined at a given DOA as

$$
\mathbf{g}_{e}\left(\eta_{l}\right)=\left\lceil\mathbf{C}^{-1} \mathbf{a}_{e m b}\left(\eta_{l}\right)\right] \cdot / \mathbf{a}_{\text {ideal }}\left(\eta_{l}\right)
$$

With an interpolated $\hat{\mathbf{g}}_{e}(\eta)$, the array response interpolation is then computed as

$$
\hat{\mathbf{a}}_{e m b}(\eta)=\mathbf{C}\left[\mathbf{a}_{\text {ideal }}(\eta) \odot \hat{\mathbf{g}}_{e}(\eta)\right]
$$

For both (5) and (7), the array response with interpolation error $\tilde{\mathbf{a}}_{e m b}(\eta)$ can be written as

$$
\mathbf{a}_{e m b}(\eta)=\hat{\mathbf{a}}_{e m b}(\eta)+\tilde{\mathbf{a}}_{e m b}(\eta)
$$

where $\tilde{\mathbf{a}}_{e m b}(\eta)$ represents the interpolation error. We use a simple model to describe $\tilde{\mathbf{a}}_{e m b}(\eta)$ [9], [26], [28]:

$$
\begin{aligned}
& E\left[\tilde{\mathbf{a}}_{e m b}\left(\eta_{m}\right)\right]=0, \\
& E\left[\tilde{\mathbf{a}}_{e m b}\left(\eta_{m}\right) \tilde{\mathbf{a}}_{e m b}^{H}\left(\eta_{m}\right)\right]=\sigma_{a}^{2} \mathbf{I}, \\
& E\left[\tilde{\mathbf{a}}_{e m b}\left(\eta_{m}\right) \tilde{\mathbf{a}}_{e m b}^{T}\left(\eta_{m}\right)\right]=0, \\
& m=1, \cdots, M
\end{aligned}
$$


where $E[\bullet]$ is statistical expectation and $(\bullet)^{H}$ denotes complex conjugate and transpose, so that the statistics of each element error under this model is independent of the DOA $\eta$. We will later use the simple model (9) to predict the performance of DOA estimation methods when using an interpolated array model, assuming that the error variance is small.

The idea is now that the correction vector $\mathbf{g}(\eta)$ in the first array response decomposition model in (2) is much smoother than the array response $\mathbf{a}_{e m b}(\eta)$, since parts of the phase variation in one embedded element is due to the ideal array response vector $\mathbf{a}_{\text {ideal }}(\eta)$. In an ideal uniform antenna array model, the array response $\mathbf{a}_{e m b}(\eta)$ can be reduced to $\mathbf{a}_{e m b}(\eta)=\mathbf{a}_{\text {ideal }}(\eta)$. Then, in the first model the resulting correction vector $\mathbf{g}(\eta)$ is $\mathbf{g}(\eta)=\lceil 1, \cdots, 1\rceil^{T}$. Clearly, $\mathbf{g}(\eta)$ will be much smoother than $\mathbf{a}_{e m b}(\eta)$. Further, in the second model if the mutual coupling matrix $\mathbf{C}=\mathbf{I}$, where $\mathbf{I}$ is the identity matrix, the correction vector $\mathbf{g}_{e}(\eta)$ can be reduced to $\mathbf{g}_{e}(\eta)=\mathbf{g}(\eta)$. However, in practice the mutual coupling is typically not an identity matrix. Then, $\mathbf{g}_{e}(\eta)$ will be smoother than $\mathbf{g}(\eta)$ when $\mathbf{C} \neq \mathbf{I}$, since parts of the phase variation is due to the mutual coupling matrix $\mathbf{C}$ and the ideal array response vector $\mathbf{a}_{\text {ideal }}(\eta)$. Numerical results of smoothness, for a real antenna array, are presented in Section 6.

\section{DOA estimation using MUSIC}

There are many different algorithms to estimate DOA using the array response. Here, we will focus on the MUSIC algorithm, which is based on the second-order properties of $\mathbf{X}(k)$ [1], [34]-[35]. Assuming the noise and signals are uncorrelated, in (1), we have the spatial covariance matrix

$$
\begin{aligned}
\hat{\mathbf{R}}_{x x} & =\lim _{K \rightarrow \infty} \frac{1}{K} \sum_{k=1}^{K} \mathbf{X}(k) \mathbf{X}(k)^{H}=\mathbf{R}_{x x} \\
& =\mathbf{A P A}^{H}+\sigma^{2} \mathbf{I}
\end{aligned}
$$

where $\mathbf{A}=\left[\mathbf{a}_{e m b}\left(\eta_{1}\right), \cdots, \mathbf{a}_{e m b}\left(\eta_{M}\right)\right]$ is the true array response at the angles of arrival $\eta_{1}, \cdots, \eta_{M}$, $\mathbf{P}$ is the covariance matrix of the signals and $\sigma^{2}$ is the noise power. It is easily shown that the eigendecomposition of $\mathbf{R}_{x x}$ can be expressed as

$$
\mathbf{R}_{x x}=\sum_{i=1}^{N} \gamma_{i} \mathbf{e}_{i} \mathbf{e}_{i}^{H}=\mathbf{E}_{s} \Lambda_{s} \mathbf{E}_{s}^{H}+\sigma^{2} \mathbf{E}_{n} \mathbf{E}_{n}^{H}
$$

where the signal subspace matrix $\mathbf{E}_{s}=\left[\mathbf{e}_{1}, \cdots, \mathbf{e}_{M}\right]$ contains the $M$ principal eigenvectors, $\Lambda_{s}=\operatorname{diag}\left\{\gamma_{1}, \gamma_{2}, \cdots, \gamma_{M}\right\}$, and the noise subspace matrix $\mathbf{E}_{n}=\left\lceil\mathbf{e}_{M+1}, \cdots, \mathbf{e}_{N}\right\rceil$ is spanned by 
the noise eigenvectors, $\gamma_{1} \geq \cdots \geq \gamma_{M} \geq \gamma_{M+1}=\cdots=\gamma_{N}=\sigma^{2}$. The noise subspace eigenvectors are orthogonal to the array response at the angles of arrival. In the MUSIC algorithm, the last relation is exploited by forming a pseudo-spectrum

$$
P_{M U}(\eta)=\frac{\left\|\mathbf{a}_{e m b}(\eta)\right\|^{2}}{\left\|\mathbf{E}_{n}^{H} \mathbf{a}_{e m b}(\eta)\right\|^{2}}
$$

The MUSIC DOA estimates are now the locations of the $M$ highest peaks of $P_{M U}(\eta)$.

Under the model of (9), for known and small variance $\sigma_{a}^{2}$ of the array response error $\tilde{\mathbf{a}}_{e m b}(\eta)$, Swindlehurst and Kailath have shown [5] that the theoretical covariance matrix of the MUSIC estimates is given by

$$
\begin{aligned}
\mathbf{C}_{M U} & =E\left[(\hat{\eta}-\eta)(\hat{\eta}-\eta)^{T}\right] \\
& =\frac{\sigma_{a}^{2}}{2}\left(\mathbf{D}^{H} \mathbf{P}_{A}^{\perp} \mathbf{D} \odot \mathbf{I}\right)^{-1}
\end{aligned}
$$

where the definitions

$$
\begin{aligned}
\mathbf{D} & =\left[\frac{\partial \mathbf{a}_{e m b}\left(\eta_{1}\right)}{\partial \eta_{1}}, \cdots, \frac{\partial \mathbf{a}_{e m b}\left(\eta_{M}\right)}{\partial \eta_{M}}\right] \\
\mathbf{P}_{A}^{\perp} & =\left\lceil\mathbf{I}-\mathbf{A}\left(\mathbf{A}^{H} \mathbf{A}\right)^{-1} \mathbf{A}^{H}\right\rceil=\mathbf{E}_{n} \mathbf{E}_{n}^{H} .
\end{aligned}
$$

are used.

\section{Interpolation methods}

\subsection{Interpolation 1: The direct interpolation of $\mathrm{a}_{e m b}(\eta)$}

We assume that the array response is accurately measured at a set of DOAs $\phi_{l}, l=1,2, \cdots, L$. Here, we will present the interpolation of the array response for a 1-D case (azimuth angle $\phi$ ) estimation problem. The most direct approach is to interpolate the real and imaginary parts of the $N$ elements of the array response to any desired DOA using local linear interpolation (LLI) [27]-[28]. Then, for LLI in the interval $\left(\phi_{l}, \phi_{l+1}\right)$, the estimated error of the $n$-th element response can be bounded by

$$
\begin{aligned}
&\left|\hat{a}_{e m b, n}(\phi)-a_{e m b, n}(\phi)\right| \leq \frac{\left|\phi_{l+1}-\phi_{l}\right|^{2}}{8} \times \\
& \max _{\phi \in\left(\phi_{l}, \phi_{l+1}\right)}\left|\frac{\partial^{2} a_{e m b, n}(\phi)}{\partial \phi^{2}}\right|
\end{aligned}
$$

where $a_{e m b, n}(\phi)$ is the $n$-th element response, $\phi_{l}$ is the measured point, and $\left|\phi_{l+1}-\phi_{l}\right|$ is the grid size of the interpolation, for $l=1,2, \cdots, L-1$. This shows clearly that the interpolated response 
is degraded when the true response is not a smooth function of the DOA. In a real antenna array, the important problem is that the function of $\mathbf{a}_{e m b}(\phi)$ is difficult to model without any errors.

For a fixed polar angle $\theta$, clearly $a_{e m b, n}(\phi)$ is a $2 \pi$-periodic function, and can be expressed in terms of a Fourier series expansion as

$$
a_{e m b, n}(\phi)=\mathbf{h v}(\phi)+e(\phi)
$$

where $\mathbf{h}$ is the effective aperture distribution function (EADF) vector, which can be computed by an Inverse Discrete Fourier Transform (IDFT) of the uniform grid points $\left\{a_{e m b, n}\left(\phi_{1}\right), \cdots, a_{e m b, n}\left(\phi_{L}\right)\right\}$ covering the whole range $(0,2 \pi)$, and $\mathbf{v}(\phi)$ is a Fourier vector (Vandermonde structured vector) [24], $[26]$.

$$
\mathbf{v}(\phi)=\left[e^{-j \frac{L-1}{2} \phi}, e^{-j \frac{L-3}{2} \phi}, \cdots, 1, e^{j \phi}, \cdots, e^{j \frac{L-1}{2} \phi}\right]^{T}
$$

The upper bound of estimated error in (14) using the second derivative of $a_{\text {emb }, n}(\phi)$ is approximated by the formula with the second derivative of $\mathbf{v}(\phi)$.

$$
\begin{aligned}
& \left|\hat{a}_{e m b, n}(\phi)-a_{e m b, n}(\phi)\right| \\
& \quad \lesssim \frac{\left|\phi_{l+1}-\phi_{l}\right|^{2}}{8} \max _{\phi \in\left(\phi_{l}, \phi_{l+1}\right)}\left|\mathbf{h} \frac{\partial^{2} \mathbf{v}(\phi)}{\partial \phi^{2}}\right| \\
& \quad=\frac{\left|\phi_{l+1}-\phi_{l}\right|^{2}}{8} \max _{\phi \in\left(\phi_{l}, \phi_{l+1}\right)}\left|\mathbf{a}_{e m b, n} \mathbf{V}^{-1} \frac{\partial^{2} \mathbf{v}(\phi)}{\partial \phi^{2}}\right|
\end{aligned}
$$

where $a_{e m b, n}(\phi)$ is the $n$-th element sample vector $\left\{a_{e m b, n}\left(\phi_{l}\right)\right\}_{l=1}^{L}$ and $\mathbf{V}$ is the $L \times L$ Vandermode matrix $\left\{\mathbf{v}\left(\phi_{l}\right)\right\}_{l=1}^{L}$. Strictly speaking, (17) is not guaranteed to be an upper bound anymore, but it is a good approximation if $T$, is large enough. It is clear that the error depends on the grid size $\left|\phi_{l+1}-\phi_{l}\right|$ and the $n$-th element response $a_{e m b, n}(\phi)$.

A truncated model could be used, where $Q(Q \leq L)$ is the number of truncated Fourier terms which is odd and $I$, is the number of grid points. Observe that the error in the Fourier-based interpolation model depends on the $n$-th element response $a_{e m b, n}(\phi)$ and the number of truncated Fourier terms $L$ [24]-[26]. In addition, the implementation of the array response can be based on 2D FFT [25], [36]-[37]. The Fourier-based interpolation is globally valid, whereas the LLI gives local models that together constitute a global response model.

\subsection{Interpolation 2: The interpolation of correction vector $\mathbf{g}(\eta)$}

At a fixed $\theta$, the correction vector $\mathbf{g}(\phi)$ can be determined by (4) in the first model using the 
known array response $\mathbf{a}_{e m b}(\phi)$ at a given DOA. Since the correction vector $\mathbf{g}(\phi)$ is potentially a much smoother function of the DOAs than $\mathbf{a}_{e m b}(\phi)$, it is natural to try linear interpolation. Combing (4), (5) and (15), the error of the $n$-th element estimated response using the LLI method can be bounded by

$$
\begin{aligned}
& \left|\hat{a}_{e m b, n}(\phi)-a_{e m b, n}(\phi)\right| \\
& \quad \lesssim \frac{\left|\phi_{l+1}-\phi_{l}\right|^{2}}{8} \max _{\phi \in\left(\phi_{l}, \phi_{l+1}\right)}\left|\mathbf{g}_{n} \mathbf{V}^{-1} \frac{\partial^{2} \mathbf{v}(\phi)}{\partial \phi^{2}}\right|
\end{aligned}
$$

where $\mathbf{g}_{n}$ is the $n$-th element sample vector $\left\{g_{n}\left(\phi_{l}\right)\right\}_{l=1}^{L}$. The error clearly depends on the interpolation grid size and the $n$-th element of the correction vector $\mathbf{g}(\phi)$.

In (4), like $\mathbf{a}_{e m b}(\phi)$, the correction vector $\mathbf{g}(\phi)$ also represents a $2 \pi$-periodic function in $\phi$ for a fixed polar angle $\theta$, which can be interpolated by (15). Using (5), the array response estimate $\hat{\mathbf{a}}_{e m b}(\phi)$ can be computed. The error also depends on the correction vector $\mathbf{g}(\phi)$ and the number of truncated Fourier terms $Q$.

\subsection{Interpolation 3: The interpolation of correction vector $\mathrm{g}_{e}(\eta)$}

\subsubsection{The mutual coupling matrix is known}

Assuming the mutual coupling matrix is known, the correction vector $\mathbf{g}_{e}(\phi)$ can be determined by (6) in the second model using the known array response $\mathbf{a}_{e m b}(\phi)$. The correction vector $\mathbf{g}_{e}(\phi)$ will be much smoother than the correction vector $\mathbf{g}(\phi)$, provided that $\mathbf{C}$ captures parts of the phase dependence from the mutual coupling. The LLI can also be used to interpolate the real and imaginary parts of $\mathbf{g}_{e}(\phi)$. The $n$-th element of the correction vector $\mathbf{g}_{e}(\phi)$ interpolation error using the LLI method can then be bounded by

$$
\begin{aligned}
& \left|\hat{g}_{e, n}(\phi)-g_{e, n}(\phi)\right| \\
& \quad \lesssim \frac{\left|\phi_{l+1}-\phi_{l}\right|^{2}}{8} \max _{\phi \in\left(\phi_{l}, \phi_{l+1}\right)}\left|\mathbf{g}_{e, n} \mathbf{V}^{-1} \frac{\partial^{2} \mathbf{v}(\phi)}{\partial \phi^{2}}\right|
\end{aligned}
$$

where $\mathbf{g}_{e, n}$ is the $n$-th element sample vector $\left\{g_{e, n}\left(\phi_{l}\right)\right\}_{l=1}^{L}$. Under (3) and (7), the upper bound error of the $n$-th element response may be written as

$$
\begin{aligned}
& \left|\hat{a}_{e m b, n}(\phi)-a_{e m b, n}(\phi)\right| \\
& \quad \lesssim\left|\mathbf{C}_{r, n} \mathbf{a}_{\text {ideal }}(\phi)\right| \max _{n \in(l, N)}\left|\hat{g}_{e, n}(\phi)-g_{e, n}(\phi)\right|
\end{aligned}
$$

where $\mathbf{C}_{r, n}=\left[c_{n, 1}, \cdots, c_{n, N}\right]$ is the $n$-th row of the mutual coupling matrix $\mathbf{C}$. The error is clearly due to the grid size, the mutual coupling matrix $\mathbf{C}$, the ideal array steering vector $\mathbf{a}_{\text {ideal }}(\phi)$ and the 9 
correction vector $\mathbf{g}_{e}(\phi)$.

Like $\mathbf{g}(\phi)$, the correction vector $\mathbf{g}_{e}(\phi)$ is also a $2 \pi$-periodic function. Thus, the Fourier-based interpolation approach can be used to interpolate $\mathbf{g}_{e}(\phi)$. Consequently, the array response estimate $\hat{\mathbf{a}}_{e m b}(\phi)$ can be computed by (7). The error also depends on the correction vector $\mathbf{g}_{e}(\phi)$ and the number of truncated Fourier terms $Q$.

If $\mathbf{C}=\mathbf{I}$, the interpolation method 3 achieves the same performance as the interpolation method 2, either using LLI or using Fourier-based interpolation. In practice, the function of $\mathbf{a}_{e m b}(\phi)$ and the mutual coupling matrix $\mathbf{C}$ are determined by the properties of the real antenna array, such as array structure and the antenna elements.

\subsubsection{Estimation of the mutual coupling matrix}

In most mutual coupling compensation techniques, a matrix is used to encapsulate the effect of mutual coupling as well as the amplitude and phase distortions caused by imperfect antenna array elements [35], [38]-[39]. In the second model, the correction vector $\mathbf{g}_{e}(\eta)$ can be obtained by measuring each isolated element response in the lab, since $\mathbf{g}_{e}(\eta)$ only contains the properties of the isolated antenna element. Then, we can estimate mutual coupling $\mathbf{C}$ from the second model.

Assume that there are $L(L>N)$ isolated element response data and array response data measured in a lab (or generated from an EM simulation program). In the second model, the optimal mutual coupling matrix $\mathbf{C}$ is then determined using least-squares as

$$
\hat{\mathbf{C}}=\arg \min _{\mathbf{C}}\left\|\mathbf{A}_{e m b}-\mathbf{C}\left[\mathbf{A}_{\text {ideal }} \odot \mathbf{G}_{e}\right]\right\|_{F}^{2}
$$

where the subscript $F$ means the Frobenius norm, $\mathbf{A}_{e m b}=\left[\mathbf{a}_{e m b}\left(\eta_{1}\right), \cdots, \mathbf{a}_{e m b}\left(\eta_{L}\right)\right]$ is a matrix of array response at $L$ points, $\mathbf{G}_{e}=\left[\mathbf{g}_{e}\left(\eta_{1}\right), \cdots, \mathbf{g}_{e}\left(\eta_{L}\right)\right]$ is a matrix of correction vectors (measured isolated element response in a lab) at $L$ points, and $\mathbf{A}_{\text {ideal }}$ is a matrix with the corresponding ideal steering vectors. If the mutual coupling matrix $\mathbf{C}$ is a full matrix with no special structure, the solution is

$$
\left.\hat{\mathbf{C}}=\mathbf{A}_{\text {emb }}\left\lceil\mathbf{A}_{\text {ideal }} \odot \mathbf{G}_{e}\right]^{H}\left(\left[\mathbf{A}_{\text {ideal }} \odot \mathbf{G}_{e}\right\rceil \mid \mathbf{A}_{\text {ideal }} \odot \mathbf{G}_{e}\right]^{H}\right)^{-1}
$$

when $t \rightarrow \infty, \hat{\mathbf{C}}$ is a perfect estimate of $\mathbf{C}$. Normally, the minimum value of the criterion in (22) is not zero when $L>N$, because (3) is never perfect. Hence, we update the estimated correction vector $\tilde{\mathbf{g}}_{e}(\eta)$ at given DOAs by 


$$
\tilde{\mathbf{g}}_{e}(\eta)=\mathbf{a}_{e m b}(\eta) \cdot /\left[\hat{\mathbf{C}} \mathbf{a}_{i d e a l}(\eta)\right]
$$

where $\hat{\mathbf{C}}$ is the estimate from (22). Consequently, the array response interpolation $\hat{\mathbf{a}}_{e m b}(\eta)$ can be expressed as

$$
\hat{\mathbf{a}}_{e m b}(\eta)=\hat{\mathbf{C}}\left\lceil\mathbf{a}_{i d e a l}(\eta) \odot \hat{\tilde{\mathbf{g}}}_{e}(\eta)\right\rceil
$$

where $\hat{\tilde{\mathbf{g}}}_{\rho}(\eta)$ is the interpolated estimated correction vector $\tilde{\mathbf{g}}_{e}(\eta)$.

\section{The real Array of 12 quadrifilar helix antennas}

The LEO satellite tests mobile communication technology with a circular array which is composed of 12 identical quadrifilar helix antennas (QHAs), with respect to rotational symmetry about the origin [40]. The first element is fixed in x-axis, and the other elements are located clockwise in the x-y plane. The uplink signal wavelength is $\lambda=150 \mathrm{~mm}$ and the array radius is $r=150 \mathrm{~mm}$. Thus, the ideal array response vector can be calculated by

$$
\mathbf{a}_{\text {ideal }}(\theta, \phi)=\left[\begin{array}{c}
\exp \left(j \frac{2 \pi}{\lambda} r \sin \theta \cos \left(\phi-\frac{2 \pi \cdot 0}{N}\right)\right) \\
\exp \left(j \frac{2 \pi}{\lambda} r \sin \theta \cos \left(\phi-\frac{2 \pi \cdot 1}{N}\right)\right) \\
\vdots \\
\exp \left(j \frac{2 \pi}{\lambda} r \sin \theta \cos \left(\phi-\frac{2 \pi \cdot(N-1)}{N}\right)\right)
\end{array}\right]
$$

where $N=12$.

Actually, the accurately measured array response and isolated element response in the lab can be replaced by an electromagnetic simulation as the hardware error and measurement error will then vanish. In our paper, we use the simulated far-field pattern by the CST Microwave Studio software, which is based on the finite integration technique (FIT), instead of the measured lab data.

Fig. 1 represents the element components of the first isolated QHA and its far-field pattern of amplitude and phase. The isolated element pattern (isolated element response) is due to the properties of the isolated antenna element and is not omnidirectional. Fig. 2 represents the circular antenna array and the far-field pattern of the first embedded element (the first element of array response), which is obtained by exciting the first element while all other elements are terminated in their own characteristic impedance [41], [42]. In this antenna array, not only the isolated element patterns are the same (rotated by $2 \pi / N)$, but also the embedded element patterns are identical due to symmetry: 


$$
a_{e m b, n}(\theta, \phi)=a_{e m b, 1}\left(\theta, \phi-(n-1) \frac{2 \pi}{N}\right)
$$

(a)

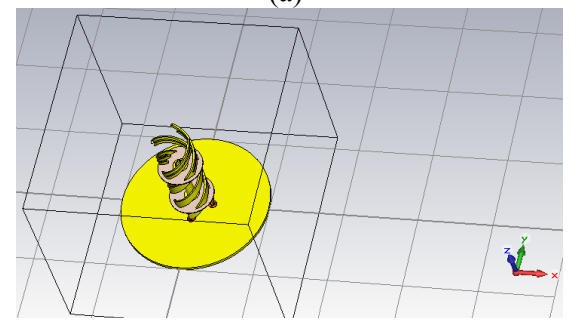

(b)
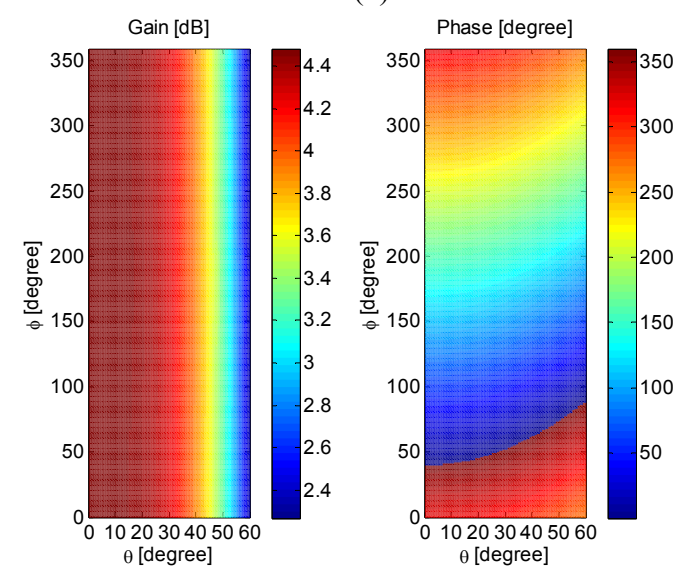

Fig. 1. The isolated QHA. (a) represents the element components. (b) shows the far-field pattern.

(a)

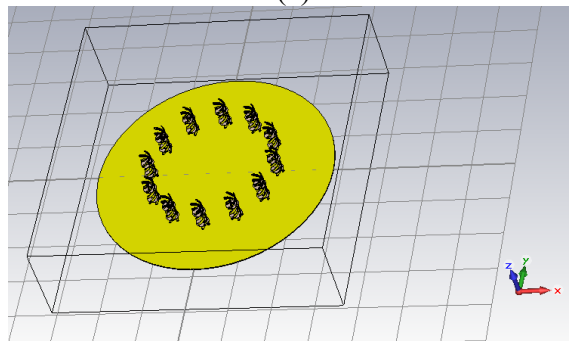

(b)
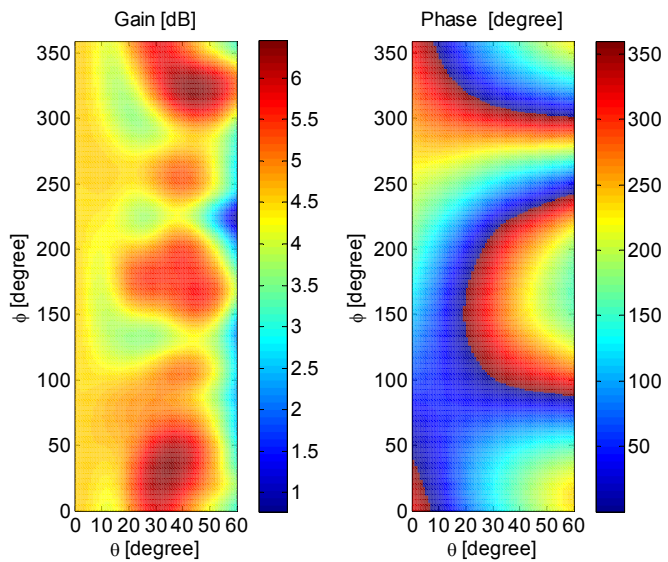

Fig. 2. The circular antenna array. (a) shows the array geometry. (b) shows the far-field pattern of the first embedded element 


\section{Interpolation results}

With the real antenna array, this section shows the smoothness measure of the three methods in Section IV. According to symmetry of the antenna array, this section just compares the interpolation results for one of the elements. Our antenna array simulation uses 1 degree grid size in both $\theta$ and $\phi$. Thus, we have $L=360$ and we use a fixed polar angle $\theta$, for $\phi=0^{\circ}, 1^{\circ}, \cdots, 359^{\circ}$.

The precision of the interpolation can be described with the average root mean square error (RMSE)

$$
R M S E=\sqrt{\frac{1}{I \cdot J} \sum_{i=1}^{I} \sum_{j=1}^{J}\left\|a\left(\theta_{i}, \phi_{j}\right)-\hat{a}\left(\theta_{i}, \phi_{j}\right)\right\|^{2}}
$$

where $I$ is the total number of test polar angles $\theta, . J$ is the total number of test azimuth angles $\phi$ in each $\theta, a\left(\theta_{i}, \phi_{j}\right)$ is the actual value, and $\hat{a}\left(\theta_{i}, \phi_{j}\right)$ is the estimated value. The resulting RMSEs of the three interpolation methods for one of the elements are shown in Fig.3.

Further, the upper bound of the LLI error can be predicted by (17), (18) and (20), respectively, which is shown as the solid line in Fig. 3 (a). It clearly shows that the upper bound of $\left|\hat{a}_{e m b, n}(\phi)-a_{e m b, n}(\phi)\right|$ in Method 3 is the smallest, since the correction vector $\tilde{\mathbf{g}}_{e}(\theta, \phi)$ is much smoother than the correction vector $\mathbf{g}(\theta, \phi)$ and the array response $\mathbf{a}_{e m b}(\theta, \phi)$. For increasing grid size of LLI, the array response estimation error of the three methods is increasing. For smaller grid size, the three methods give almost the same performance when the calibration measurements are noisy. In Fig. 3 (b), similarly, for increasing number of Fourier terms, the array response estimation error of the three methods with Fourier-based interpolation is decreasing. The error floor is determined by the measurement noise error level. Cleary, there is a link between the measurement noise power and the number of Fourier terms $Q$. When $Q \geq 45, Q \geq 25$ and $Q \geq 15$, respectively, the three methods reach the same RMSE. Another aspect of interest is the computational complexity of the three interpolation methods. Clearly, the computational complexity of Method 3 is the largest. With a smaller grid size or a larger Fourier terms, the three methods perform close to the low bound. Due to the increased complexity, Method 1 is of interest as a good trade-off between cost and accuracy.

Either for a given smaller grid size $\left|\phi_{l+1}-\phi_{l}\right|$, or for a given larger number of Fourier terms $Q$, it is obvious that interpolation method 3 performs the best, which is to be expected. Also, it is shows that the correction vector $\tilde{\mathbf{g}}_{e}(\theta, \phi)$ of interpolation method 3 is much smoother than the correction vector $\mathbf{g}(\theta, \phi)$ of interpolation method 2, and $\mathbf{g}(\theta, \phi)$ is much smoother than the array response 
$\mathbf{a}_{e m b}(\theta, \phi)$ of interpolation method 1 . Note that in our antenna array without measurement noise, the results for the LLI method and Fourier-based interpolation are very close, when the grid size of LLI is 21 degrees and the number of Fourier terms is 11 . Hence, in this given performance, the Fourier-based interpolation requires least memory, since the Fourier-based interpolation needs to store $Q=11$ numerical values for Fourier terms, as compared to 17 (calculated by 360/21) numerical values for measured points in the LLI method. The general conclusion is that the correct application of array geometry $\mathbf{a}_{\text {ideal }}(\theta, \phi)$ and mutual coupling knowledge can mitigate the smoothness problem in array interpolation.

(a)

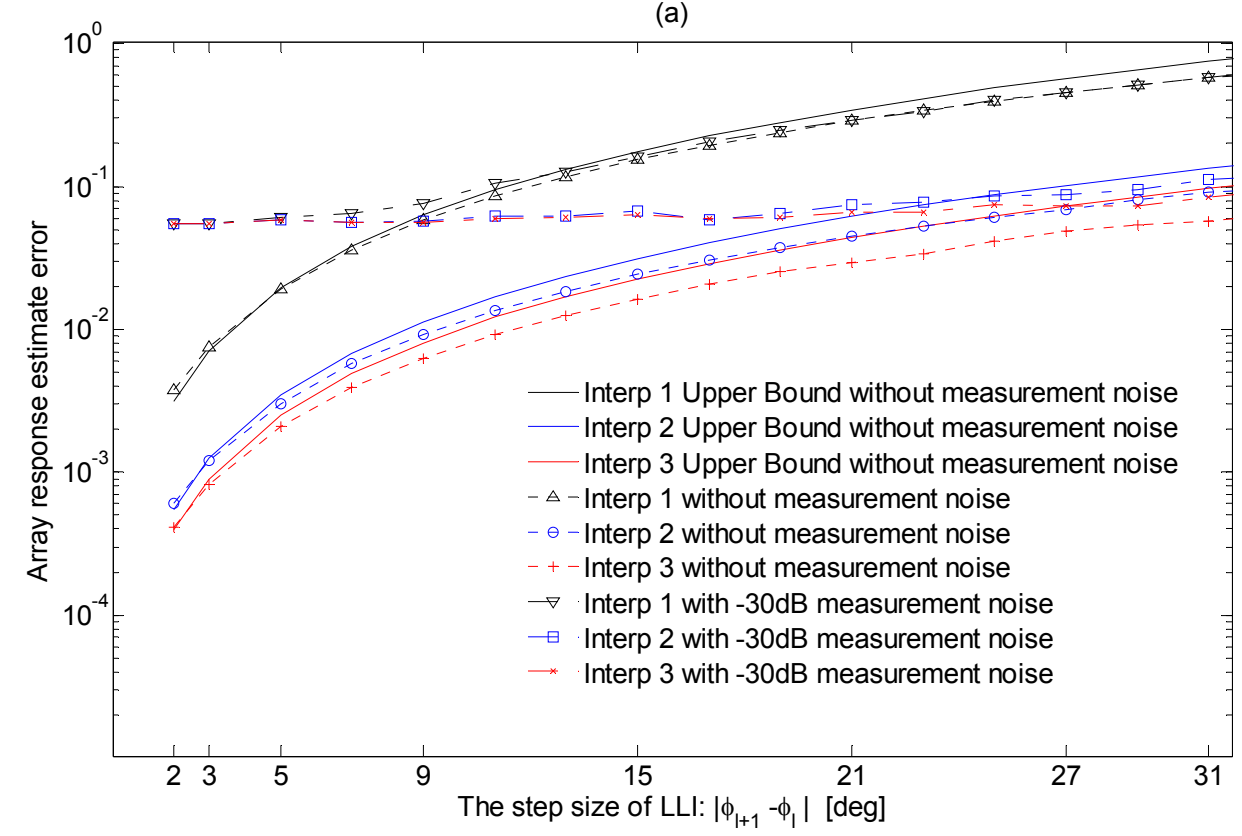

(b)

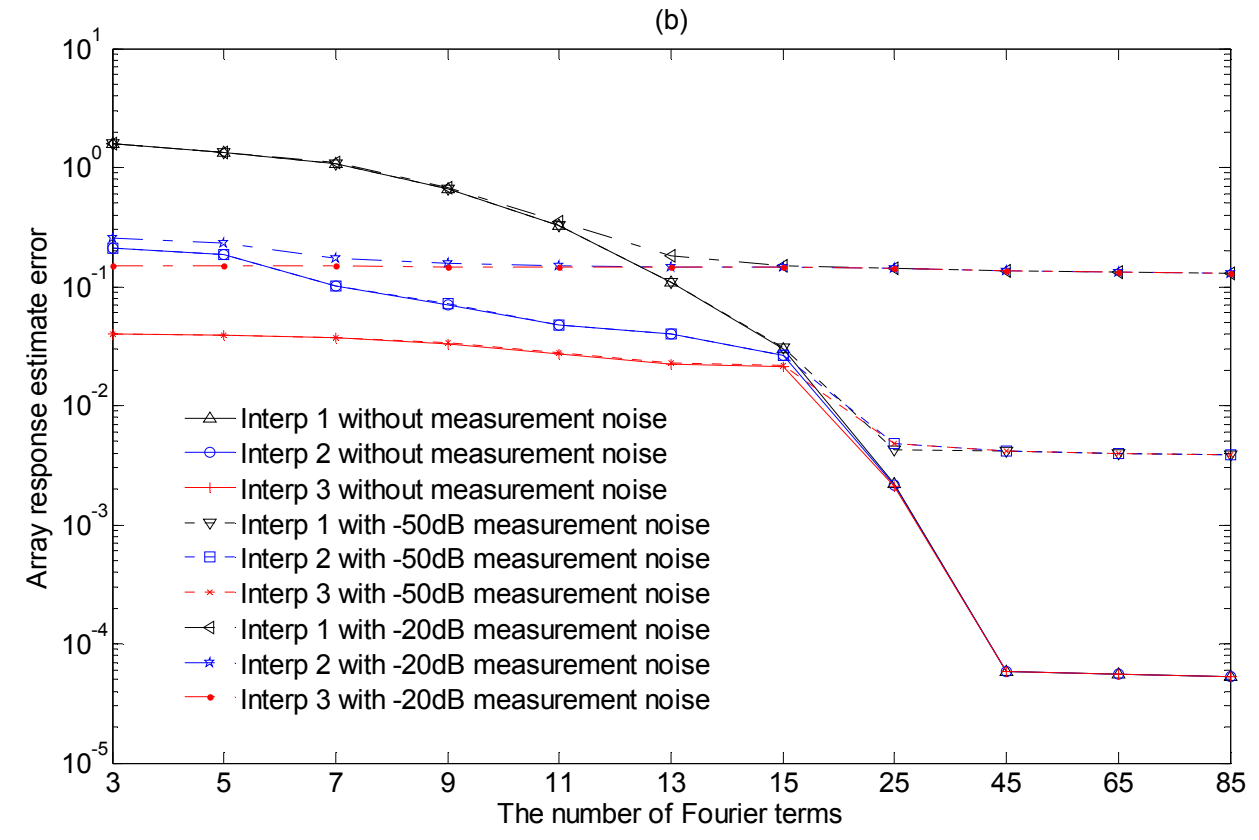


Fig. 3. Comparison between the array response interpolation and computed empirical RMSEs by using the three interpolation methods. (a) is based on the LLI. (b) is based on Fourier-based interpolation.

\section{DOA estimation results}

\subsection{Classical beamforming}

Now, we have three models of array response interpolation for classical beamforming and DOA estimation. The first model is presented in Section 4.1, which just needs the measured array response at given DOAs. The second model is explained in Section 4.2, which needs to know the elements location. The third model is presented in Section 4.3, which also needs the mutual coupling matrix. Next, we will show some examples of how the different methods affect the beam pattern, which can be calculated as

$$
P_{B F}(\theta, \phi)=\frac{\left\|\hat{\mathbf{a}}_{e m b}\left(\theta_{q}, \phi_{q}\right)^{H} \mathbf{a}_{e m b}(\theta, \phi)\right\|^{2}}{\left\|\hat{\mathbf{a}}_{e m b}\left(\theta_{q}, \phi_{q}\right)\right\|^{2}}
$$

where $\left(\theta_{q}, \phi_{q}\right)$ is the desired beam point direction.

Fig. 4 shows the beam patterns calculated at direction $\left(\theta_{q}=45^{\circ}, \phi_{q}=170^{\circ}\right)$, using the three methods. It is seen that the main lobe gains of the Interpolation 2 and 3 methods are higher than that of the Interpolation 1 method, and are close to the ideal beam gain in this scenario. In contrast, the higher side lobes of the Interpolation 1 method are due to larger errors in the interpolated array response.

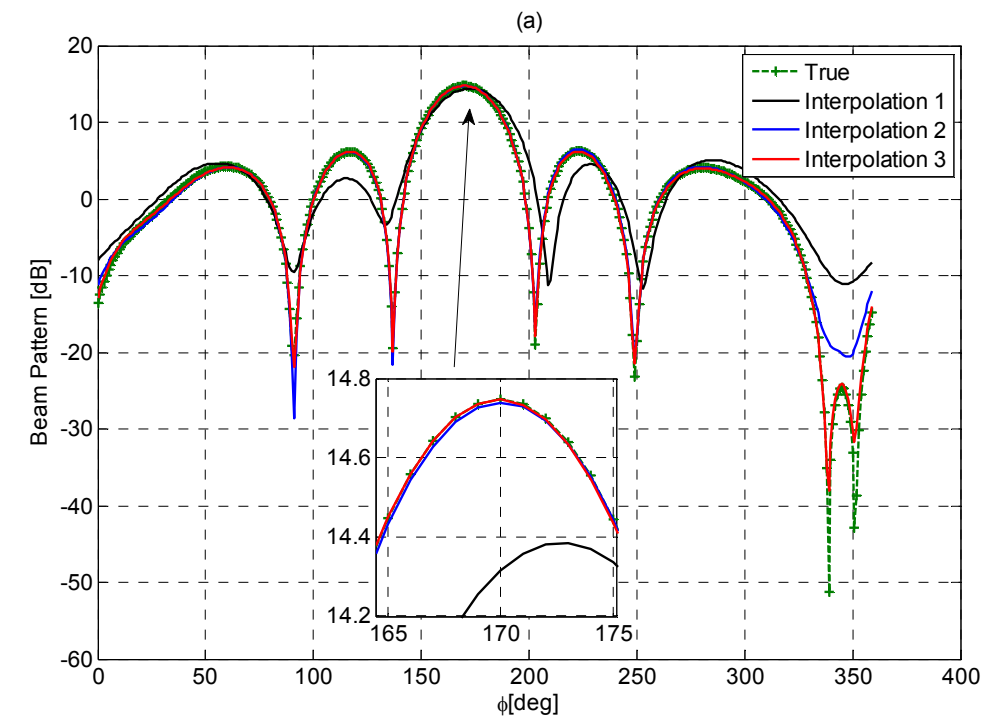




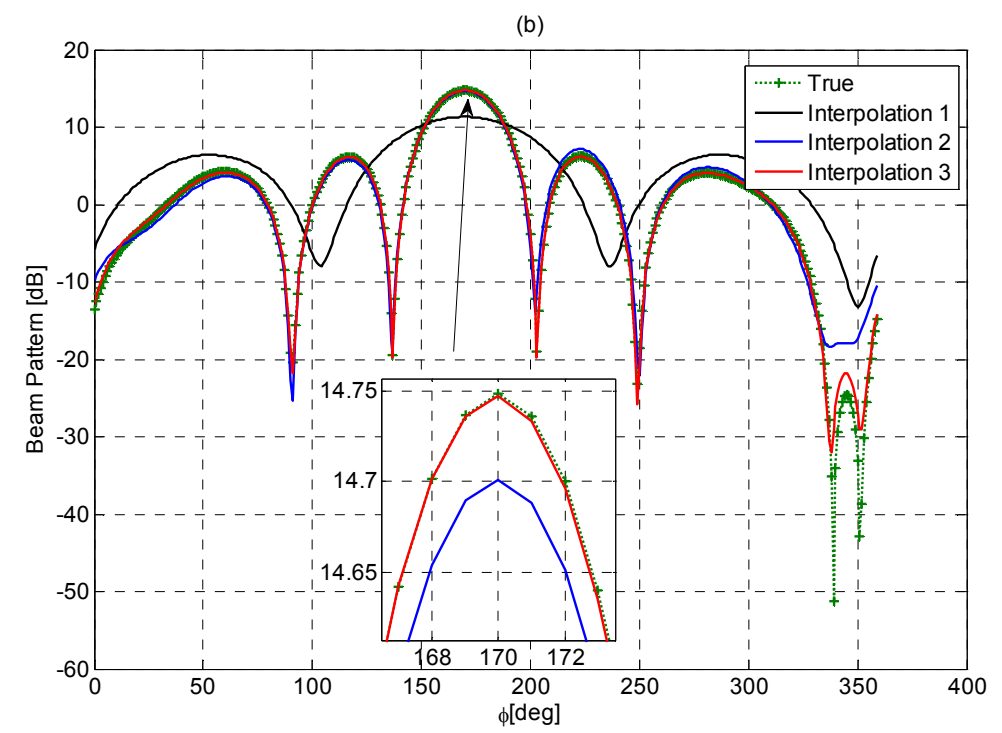

Fig. 4. Beam pattern using the three interpolation methods. In (a), the grid size of local linear interpolation is 20 degrees. In (b), the number of Fourier terms is 5.

\subsection{DOA estimation with MUSIC}

In this section, simulation examples are presented for the effect of the three methods on two closely located uncorrelated sources, where the number of snapshots is 100 and the input SNR is $10 \mathrm{~dB}$. A total of 500 trials are conducted for each example. We only show the performance of azimuth angle estimation, since the polar angle estimation presents similar results. The DOA separation $\Delta \phi$ is defined as $\Delta \phi=\left|\phi_{1}-\phi_{2}\right|$, where the polar angle is fixed at $45^{\circ}$. Cases where an algorithm fails to resolve the sources (only one local extremum within $\pm \Delta \phi$ of the true value), or where the DOA estimation error is larger than half the DOA separation, $\Delta \phi$, are declared failures, and these are not included in the RMSE calculation. If the empirical failure rate exceeds $40 \%$, the corresponding RMSE value is not included in the plot.

In the first case, the two sources are fixed at $\left(\theta_{1}=45^{\circ}, \phi_{1}=100^{\circ}\right)$ and $\left(\theta_{2}=45^{\circ}, \phi_{2}=110^{\circ}\right)$. Figure 5 shows the MUSIC pseudo-spectrum of the DOA estimation using the three interpolation methods. In Fig.5, it is clearly seen that the Interpolation 3 method shows the best performance, especially for the locations of the pseudo-spectrum peaks. In this scenario, there is only one significant peak in the MUSIC pseudo-spectrum of the Interpolation 1 method, which directly interpolates the array response, so this method fails to resolve the sources. 
(a)

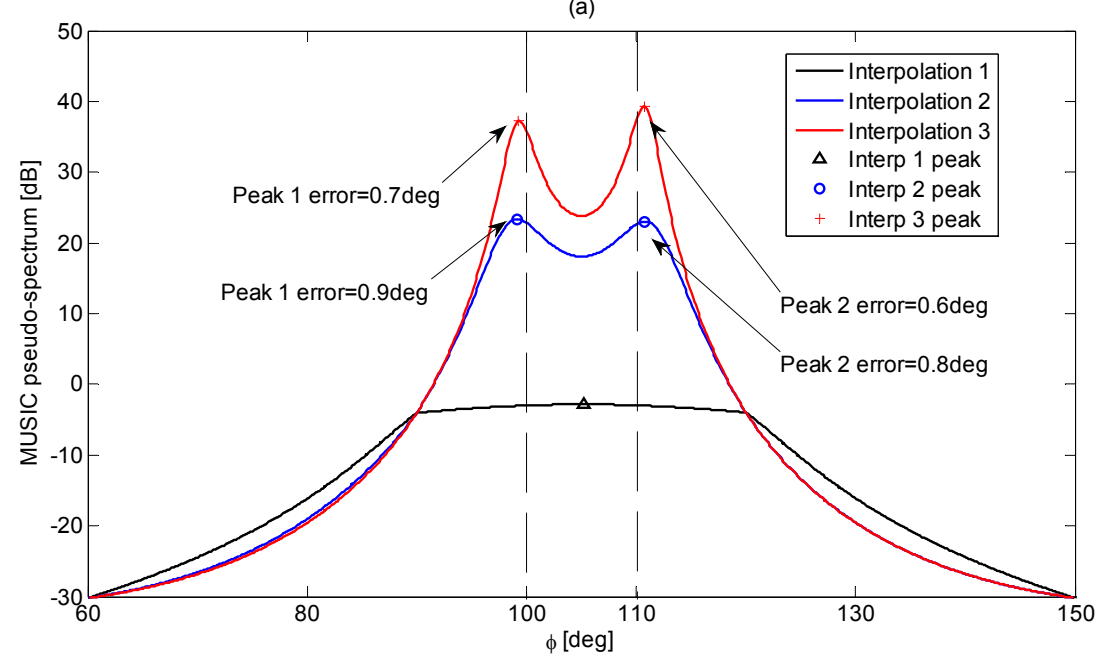

(b)

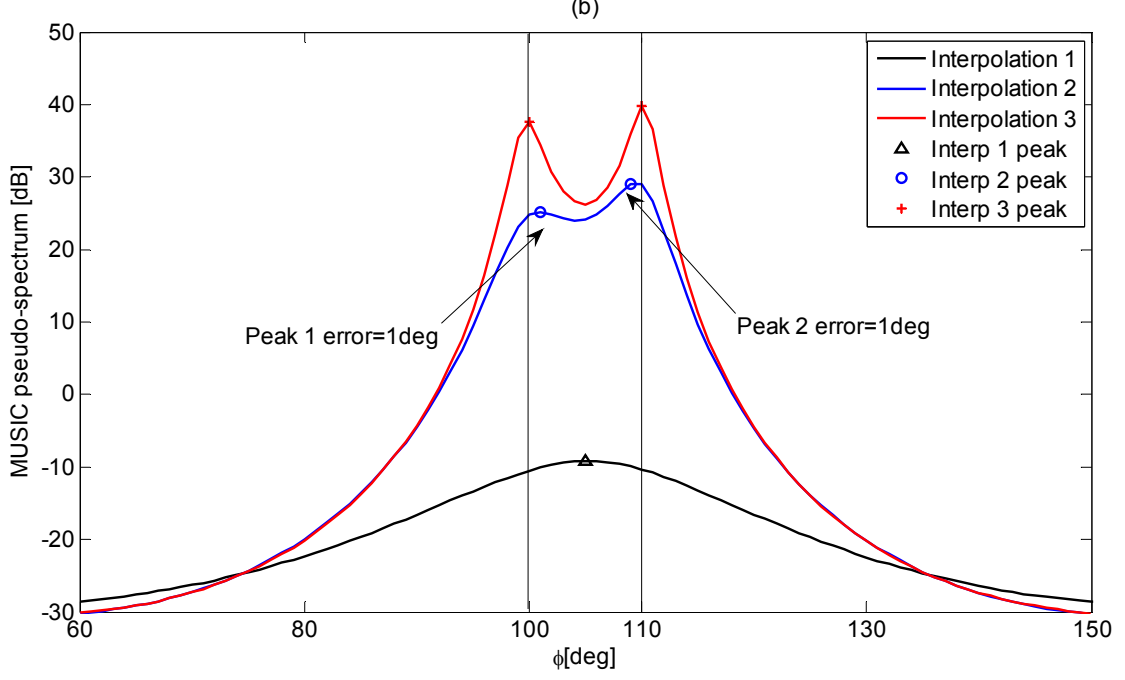

Fig. 5. MUSIC pseudo-spectrum. In (a), the grid size of local linear interpolation is 20 degrees. In (b), the number of Fourier terms is 9.

In the second case, the azimuth angle $\phi_{1}$ of the first source is fixed at $100^{\circ}$ and the second varied between $\phi_{2}=103^{\circ}$ and $\phi_{2}=160^{\circ}$. The variance of the three interpolation methods is predicted from using (13) and the performance of MUSIC with the three interpolation methods is plotted in Fig. 6 . The solid lines represent the error predicted by (13), and the dashed lines represent the empirical resulting RMSE of the three interpolation methods. In Fig. 6, (a) shows the result of LLI and (b) plots the result of Fourier-based interpolation. Note that the errors predicted by (13) match the empirical results good in both LLI and Fourier-based interpolation. It is clearly seen that the three methods ameliorate significantly with increasing DOA separation $\Delta \phi$, and the Interpolation 3 method again shows the best performance for a given source separation. It is interesting to note that the Interpolation 1 method fails, when the source separation is smaller than the grid size $\left(\left|\phi_{l+1}-\phi_{l}\right|=9^{\circ}\right)$, as shown in Fig. 6 (a). However, the Interpolation 2 and 3 methods break down at 7 degrees and 5 degrees separation, 
respectively, in Fig. 6 (a), since the correction vectors $\mathbf{g}(\theta, \phi)$ and $\tilde{\mathbf{g}}_{e}(\theta, \phi)$ are smoother than $\mathbf{a}_{e m b}(\theta, \phi)$. Similarly, when the separation is less than 25 degrees, the Interpolation 1 method of DOA estimation fails in Fig. 6 (b). This clearly shows that the third interpolation method not only improves the DOA estimation accuracy, but also can be used in smaller separations, due to the ability to take advantage of the smoothness in $\tilde{\mathbf{g}}_{e}(\theta, \phi)$.
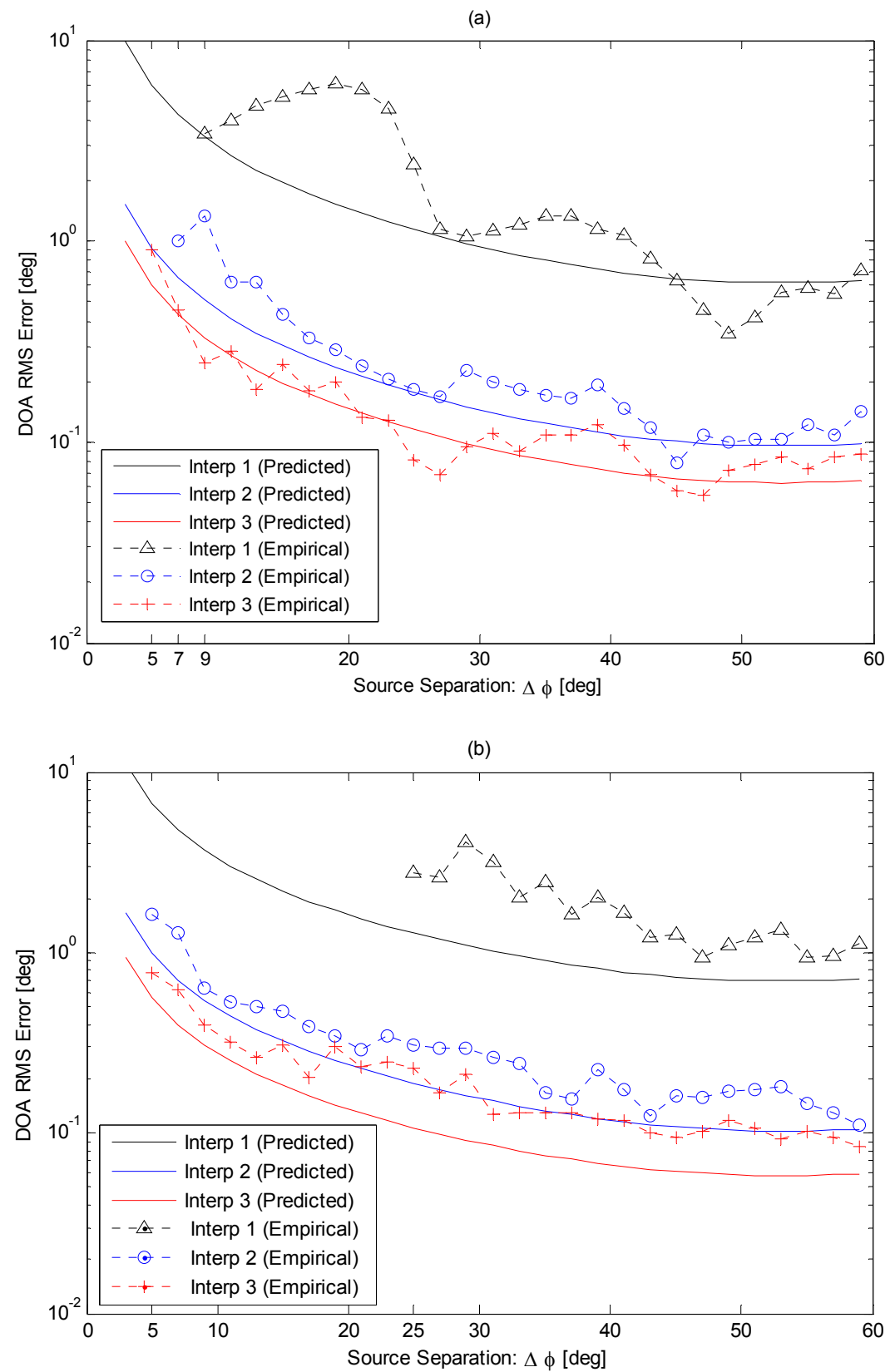

Fig. 6. Performance of the three interpolation methods versus the source separation $\Delta \phi$. (a) is based on the LLI with 21 degrees grid size. (b) is based on Fourier-based interpolation with 11 Fourier terms.

In the third case, the source separation $\Delta \phi$ is fixed at $6^{\circ}$, the azimuth angle $\phi_{1}$ of the first source 
is varied from $\phi_{1}=30^{\circ}$ to $\phi_{1}=150^{\circ}$. Fig. 7 displays the predicted and measured performance of the three interpolation methods with MUSIC versus the interpolation parameters, such as the grid size of LLI and number of Fourier terms. The solid and dashed lines correspond to that predicted by (13) and the empirical results respectively. In Fig. 7 (a), we use the LLI, where the three methods deteriorate significantly with increasing the grid size. For a given grid size of LLI, it is clearly seen that Interpolation 1 method performs the worst among the interpolation techniques in this scenario. It is also interesting to see that the Interpolation 1 method fails, when the grid size of LLI is larger than the source separation $\left(\Delta \phi=6^{\circ}\right)$, as shown in Fig. 7 (a). In contrast, when we employ the ideal array response and mutual coupling as in the Interpolation 2 and 3 methods, a large grid size of LLI can be used, where Interpolation 2 method breaks down at 20 degrees grid size and Interpolation 3 method breaks down at 33 degrees grid size. Fig. 7 (b) illustrates the performance of the three methods with Fourier-based interpolation. It is interesting to see that the three interpolation methods give consistently better results when the number of Fourier terms increases. It is clear that the number of Fourier terms can be kept as small as possible using the Interpolation 3 method with the correction vector $\tilde{\mathbf{g}}_{e}(\theta, \phi)$, which performs the best as expected.

Finally, we note that the simple model (9) gives a useful statistical characterization of the errors, so that (13) can be used to predict the DOA RMS with acceptable accuracy.

(a)

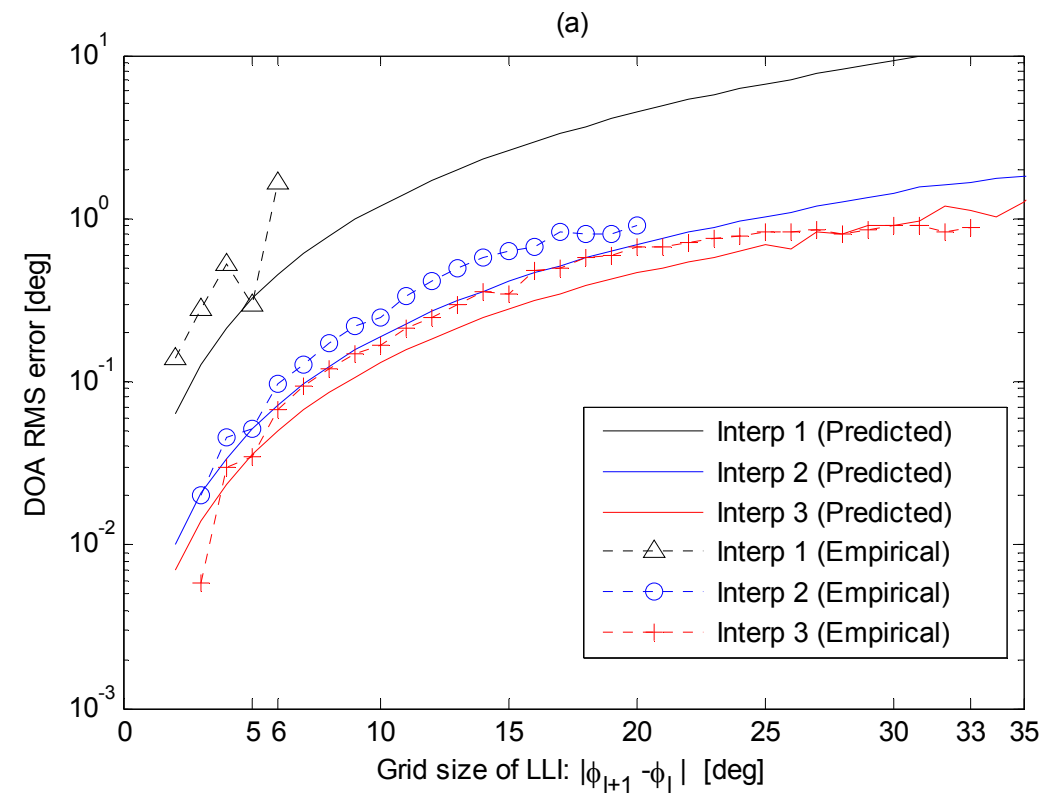




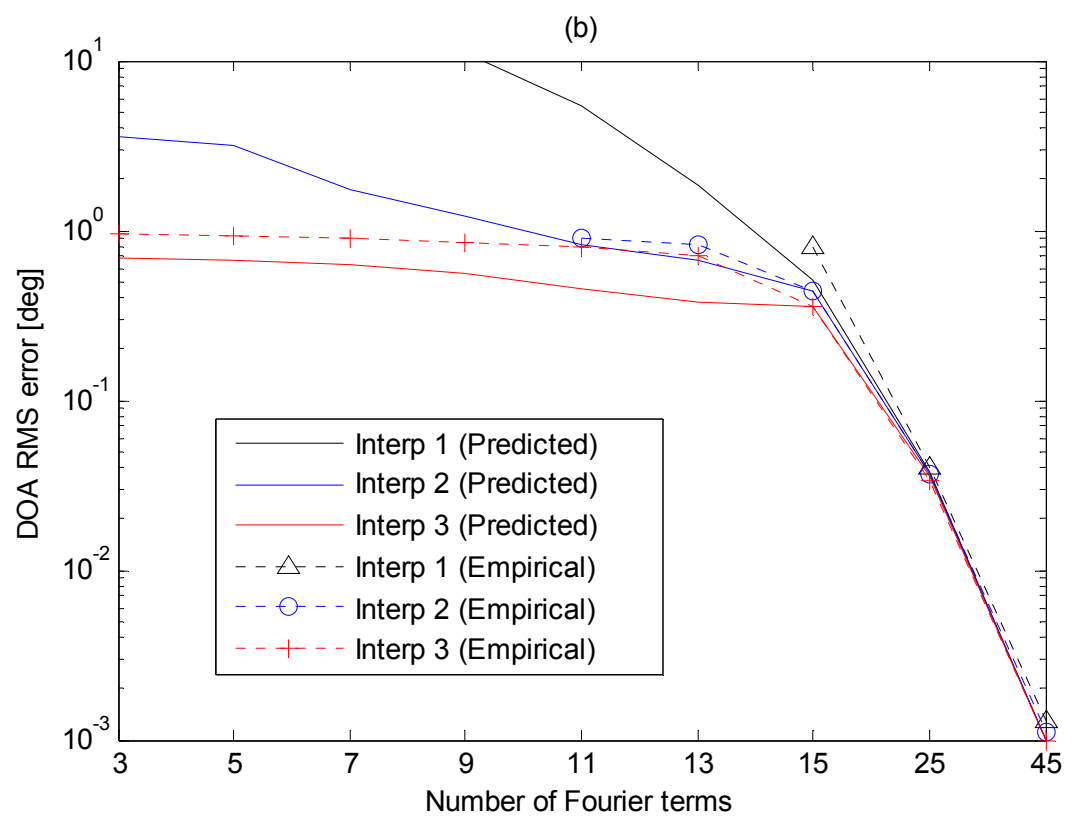

Fig. 7. Empirical RMSE of the DOA estimate versus the $\Delta \phi$ using Fourier interpolation. Performance of the three interpolation methods versus the interpolation parameters. In (a), we use the Local linear interpolation. In (b), we use the Fourier-based interpolation.

\section{Conclusion}

This paper presents a new method for array response interpolation and DOA estimation based on a correction vector interpolation and mutual coupling matrix estimation. This method combines interpolation techniques with parametric modeling. Since parts of the phase variation in one embedded element of the array response is due to the mutual coupling and the ideal array steering vector, interpolation of the correction vector yields a much more accurate model for each element separately, which is then used to update the array response.

The method is tested using a real antenna array and it is shown to improve the interpolation performance significantly. We can easily apply the interpolation of the correction vector with an estimated mutual coupling matrix and a measured array response for beamforming and arbitrary DOA estimation. The results also show that the method with correction vector interpolation and mutual coupling estimation outperforms the direct interpolation method that only uses the measured array response for a given grid. Then, the array response interpolation can be optimized by coherently combing the correction vector and the mutual coupling matrix. In our future work we plan to investigate array response interpolation and DOA estimation using measured array response data on a satellite. 


\section{References}

[1] R. Schmidt, "Multiple emitter location and signal parameter estimation," IEEE Trans. Antennas and Propagation, vol. AP-34, no. 2, pp. 276-280, Mar. 1986.

[2] R. Roy and T. Kailath, "ESPRIT—Estimation of signal parameters via rotational invariance techniques," IEEE Trans. Acoustics, Speech and Signal Processing, vol. 37, no. 7, pp. 984-995, July 1989.

[3] Z. F. Ye, J. S. Dai, X. Xu and X. P. Wu, "DOA estimation for uniform linear array with mutual coupling," IEEE Trans. Aerospace and Electronic Systems, vol. 45, no.1, pp. 280-288, Jan 2009.

[4] B. Liao, Z. G. Zhang and S. C. Chan, "DOA estimation and tracking of ULAs with mutual coupling," IEEE Trans. Aerospace and Electronic Systems, vol. 84, no. 1, pp. 891-905, Jan 2012.

[5] A. Swindlehurst and T. Kailath, "A performance analysis of subspace-based methods in the presence of model errors, Part I: The MUSIC algorithm", IEEE Trans. Signal Processing, vol.40, no. 7, pp. 1758-1774, July 1992.

[6] A. Swindlehurst and T. Kailath, "A performance analysis of subspace-based methods in the presence of model errors: Part II- Multidimensional algorithms”, IEEE Trans. signal processing, vol. SP-41, pp. 2882-2890, Sept. 1993.

[7] A. Ferréol, P. Larzabal and M. Viberg, "Performance prediction of maximum-likelihood direction-of-arrival estimation in the presence of modeling errors", IEEE Trans. Signal Processing, vol. 56, no. 10, pp. 4785-4793, Oct. 2008.

[8] A. Ferréol, P. Larzabal and M. Viberg, "Statistical analysis of the MUSIC algorithm in the presence of modeling errors, taking into account the resolution probability”, IEEE Trans. Signal Processing, vol. 58, no. 8, pp. 4156-4166, Aug. 2010.

[9] M. Jansson, A. Swindlehurst, and B. Wahlberg, "Weighted subspace fitting for general array error models," IEEE Trans. Signal Processing, vol. 46, no. 9, pp. 2484-2498, Sept. 1998.

[10] A. Flieller, A. Ferréol, P. Larzabal and H. Clergeot, "Robust bearing estimation in the presence of direction-dependent modelling errors: identifiability and treatment," in Conf. Rec. 1995 IEEE Int. Conf. Acoustics, Speech and Signal Processing, Detroit, MI, USA, May, 1995, vol. 3, pp. 1884-1887.

[11] B. P. Flanagan, K. L. Bell, "Array self-calibration with large sensor position errors," Signal Processing, vol. 81, 2001, pp.2201-2214.

[12] J. Kim, H. J. Yang, B. W. Jung, and J. Chun, "Blind calibration for a linear array with gain and phase error using independent component analysis," IEEE Antennas and Wireless Propagation Letters, vol. 9, pp.1259-1262, Jan. 2010.

[13] A. Liu, G. S. Liao, Q. Xu, and L. Ma, "An improved array-error estimation method for constellation SAR systems," IEEE Geoscience and Remote Sensing Letters, vol.9, pp.90-94, Jan. 2012.

[14] M. Moebus, H. Degenhardt and A. Zoubir, "Local array calibration using parametric modeling of position errors and a sparse calibration grid," in Workshop. 2009 IEEE/SP 15th Workshop. Statistical Signal Processing, Cardiff, UK, Aug. 31-Sep. 3, 2009, pp. 453-456.

[15] P. Heidenreich and A. M. Zoubir, "High-resolution direction finding of coherent sources in the presence of model errors using alternating projections," in Workshop. 2009 IEEE/SP 15th Workshop. Statistical Signal Processing, Cardiff, UK, Aug. 31-Sep. 3, 2009, pp. 521-524. 
[16] M. Lanne, A. Lundgren and M. Viberg, "Optimized beamforming calibration in the presence of array imperfections," in Conf. Rec. 2007 IEEE Int. Conf. Acoustics, Speech and Signal Processing, Honolulu, HI, USA, Apr, 2007, vol. 2, pp. 973-976.

[17] M. Lanne, M. Viberg and A. Lundgren, "Adaptive beamforming using calibration vectors with unknown gain and phase treatment," in Symp. Rec. 2007 IEEE Int. Symp. Antennas and Propagation, Honolulu, HI, USA, Jun, 2007, pp. 4208-4211.

[18] J. Dai, W. Xu and D Zhao, "Real-valued DOA estimation for uniform linear array with unknown mutual coupling," Signal Processing, vol. 92, 2012, pp. 2056-2065.

[19] C. Liu, Z. Ye and Y. Zhang, “Autocalibration algorithm for mutual coupling of planar array,” Signal Processing, vol. 90, 2010, pp. 784-794.

[20] L. Xiang, Z. Ye, X. Xu, C. Chang, W. Xu and Y. S. Hung, "Direction of arrival estimation for uniform circular array based on fourth-order cumulants in the presence of unknown mutual coupling," IET Microw. Antennas Propag., vol. 2, no. 3, pp. 281-287, 2008.

[21] A. J. Weiss and B. Friedlander, "Eigenstructure methods for direction finding with sensor gain and phase uncertainties", Circ. Syst. and Sig. Proc., vol. 9, no. 3, pp. 271-300, 1990.

[22] G. C. Brown, J. H. McClellan and E. J. Holder, "A phased array calibration technique using eigenstructure methods", in Conf. Rec. 1990 IEEE Int. Radar Conf., Arlington, VA, USA, May, 1990.

[23] B. Friedlander and A. J. Weiss, "Direction finding in the presence of mutual coupling", IEEE Tans. Antennas and Propagation, vol. 39, no. 3, pp. 273-284, March, 1991.

[24] M. A. Doron and E. Doron, "Wavefield modeling and array processing, Part I-Spatial sampling," IEEE Trans. Signal Processing, vol. 42, no. 10, Oct.1994, pp. 2549-2559.

[25] F. Belloni, A. Richter and V. Koivunen, "Extension of root-MUSIC to non-ULA array configurations," in Conf. Rec. 2006 IEEE Int. Conf. Acoustics, Speech and Signal Processing (ICASSP), France, May, 2006.

[26] F. Belloni, A. Richter and V. Koivunen, "DoA estimation via manifold separation for arbitrary array structures," IEEE Trans. Signal Processing, vol. 55, no. 10, Oct. 2007, pp.4800-4810.

[27] M. Lanne, A. Lundgren and M. Viberg, "Calibrating an array with scan dependent errors using a sparse grid," in Conf. Rec. 2006 Fortieth Asilomar Conf. Signals, Systems and Computers, Pacific, CA, USA, Oct, 2006, pp. 2242-2246.

[28] M. Viberg and A. Lundgren, "Array interpolation based on local polynomial approximation with application to DOA estimation using weighted MUSIC," in Conf. Rec. 2009 IEEE Int. Conf. Acoustics, Speech and Signal Processing, Taipei, Apr, 2009, pp. 2145-2148.

[29] Y. Inoue, K. Mori and H. Arai, "DOA estimation in consideration of the array element pattern," 2002 IEEE 55th Vehicular Technology Conference, 2002, vol. 2, pp. 745-748.

[30] A. Ferréol, P. Larzabal and M. Viberg, "On the asymptotic performance analysis of subspace DOA estimation in the presence of modeling errors: case of MUSIC,” IEEE Trans. Signal Processing, vol. 54, no. 3, pp. 907-920, Mar. 2006.

[31] J. Dai and Z. Ye, "Spatial smoothing for direction of arrival estimation of coherent signals in the presence of unknown mutual coupling," IET Signal Processing, vol. 5, Iss. 4, pp. 418-425, 2011.

[32] C. M. Schmid, S. Schuster, R.Feger and A. Stelzer, "On the effects of calibration errors and mutual coupling on the beam pattern of an antenna array," IEEE Trans. Antennas and Propagation, vol. 61, no. 8, pp. 4063-4072, Aug. 2013. 
[33] A. Ferréol, E. Boyer, P. Larzabal and M. Haardt, "On the introduction of an extended coupling matrix for a 2D bearing estimation with an experimental RF system," Signal Processing, vol. 87, pp.2005-2016, 2007.

[34] K. R. Dandekar, H. Ling and G. H. Xu, "Experimental study of mutual coupling compensation in smart antenna applications," IEEE Trans. Wireless Communications, vol. 1, no. 3, pp. 480-487, Jul. 2002.

[35] O. A. Oumar, M. F. Siyau and T. P. Sattar, "Comparison between MUSIC and ESPRIT direction of arrival estimation algorithms for wireless communication systems," in Conf. Rec. 2012 IEEE Int. Conf. Future Generation Communication Technology (FGCT), London, Dec, 2012, pp. 99-103.

[36] G. D. Galdo, J. Lotze, M. Landmann, and M. Haardt, "Modelling and manipulation of polarimatric antenna beam patterns via spherical harmonics," in Conf. Rec. 14th Eur. Signal Processing Conf. (EUSIPCO), Florence, Italy, Sep. 2006.

[37] M. Pralon, D. Schulz and R. S. Thoma, "Optimization of antenna arrays for 2D DoA estimation using EADF for Cramér-Rao lower bounds computation," in Conf. Rec. 2013 IEEE-APS Topical Conf. Antennas and Propagation in Wireless Communications (APWC). Torino, Italy, Sep, 2013, pp. 1429-1432.

[38] H. A. Abdallah and W. Wasylkiwskyj, "A numerical technique for calculating mutual impedance and element patterns of antenna arrays based on the characteristics of an isolated element," IEEE Trans. Antennas and Propagation, vol. 53, no. 10, pp. 3293-3299, Oct. 2005.

[39] Q. L. Huang, H. X. Zhou, J. H. Bao and X. W. Shi, "Mutual coupling calibration for microstrip antenna arrays via element pattern reconstruction method," IEEE Antennas and Wireless Propagation Letters, vol. 13, pp. 51-54, 2014.

[40] B. Yang, F. He, J. Jin, H. G. Xiong and G. H. Xu, "DOA estimation for attitude determination on communication satellites," Chinese Journal of Aeronautics, vol. 27, no. 3, pp. 670-677, 2014.

[41] D. F. Kelley, "Embedded element patterns and mutual impedance matrices in the terminated phased array environment," in Proc. 2005 IEEE Int. Symp. Antennas Propagation. Jul. 2005, vol. 3A, pp. 659-622.

[42] K. Yang, Z. Q. Zhao, Z. P. Nie, J. Ouyang and Q. H. Liu, "Synthesis of conformal phased arrays with embedded element pattern decomposition," IEEE Trans. Antennas and Propagation, vol. 59, no. 8, pp. 2882-2888, Aug. 2011. 